\title{
RATIONAL NONLINEAR CHARACTERS OF METABELIAN GROUPS
}

\author{
B. G. BASMAJI
}

\begin{abstract}
Let $G$ be a finite metabelian group with all nonlinear irreducible characters rational. Then the exponent of the commutator group $G^{\prime}$ is a prime or divides 16,24 , or 40 . If $G^{\prime}$ is also cyclic, then its order is a prime or divides 12.
\end{abstract}

1. Introduction. Let $G$ be a finite group having all characters (over the complex field) rational. Markel [6] proved that if $G$ is supersolvable then $G$ has order $|G|=2^{t} 3^{s}$. In [4] Gow proved that if $G$ is solvable then $|G|=2^{t} 3^{s} 5^{h}$, and in [7] Vishnevetskij proved similar results if $G$ is finite with some added conditions.

In this paper we assume that $G$ is a finite metabelian group having all nonlinear (absolutely) irreducible characters rational. After proving a few preliminary results we prove, in $\S 3$, that the exponent of $G^{\prime}$ is either a prime or divides 16,24 , or 40 . Assuming that $G^{\prime}$ is cyclic we prove, in $\S 4$, that $\left|G^{\prime}\right|$ is a prime or it divides 12 . In particular if all the characters of $G$ are rational, then the results of this paper imply that $|G|=2^{t} 3^{s}$.

2. Preliminary results. Let $G$ be a finite group. By a character of $G$ we shall always mean a character over the complex field, and by an irreducible character we shall always mean an absolutely irreducible character. A character $\chi$ of $G$ is rational (real) if $\chi(g)$ is rational (real) for all $g \in G$. If $K$ is a normal subgroup of $G$ and $a \in G$, then $\langle a\rangle$ and $\langle a K\rangle$ denote the cyclic subgroups of $G$ and $G / K$ respectively, and $|a|_{0}$ and $|a K|_{0}$ denote the orders of $\langle a\rangle$ and $\langle a K\rangle$ respectively. Also $\exp (G)$ denotes the exponent of $G$. If $A$ is a group that acts on a group $B$ then $\mathbf{C}_{A}(B)=\left\{x \in A \mid b^{x}=b\right.$ for all $\left.b \in B\right\}$.

LEMMA 1. Let $G$ be a finite group, $K$ a normal subgroup of $G, \chi$ a linear character of $K$ such that $\chi^{G}$ is irreducible, $L=\operatorname{ker} \chi$, and $N(L)$ the normalizer of $L$ in $G$. Then $\chi^{G}$ is real if and only if some element of $N(L)$ inverts every element of $K / L$ and $\chi^{G}$ is rational if and only if $N(L) / K \simeq \operatorname{Aut}(K / L)$, the automorphism group of $K / L$.

PROOF. Let $\chi_{1}$ be the Q-conjugate (complex conjugate) of $\chi$. Then $\operatorname{ker} \chi_{1}=L$, $\chi_{1}^{G}$ is irreducible and is Q-conjugate (complex conjugate) to $\chi^{G}$. From [3, (45.5)], $\chi_{1}^{G}=\chi^{G}$ if and only if $\chi_{1}$ and $\chi$ are $G$-conjugate. The results follow.

A somewhat deeper result concerning the realness of $\chi^{G}$ is given in [2, §2].

Now assume $G$ is a finite metabelian group, $A$ a normal subgroup of $G$ such that $A$ and $G / A$ are abelian. Let $\chi$ be a linear character of $A$ with $\operatorname{ker} \chi=L$, and $K(L)$ a subgroup of $N(L)$ containing $A$ such that $K(L) / L$ is a maximal abelian subgroup

Received by the editors November 11, 1980 and, in revised form, August 14, 1981.

1980 Mathematics Subject Classification. Primary $20 \mathrm{C15}$.

Key words and phrases. Characters, real characters, rational characters, induced characters. 
of $N(L) / L$. The $\chi$ has $|K(L) / A|$ extensions $\chi^{\prime}$ to $K(L)$ and each $\chi^{\prime G}$ is irreducible and is a constituent of $\chi^{G}$. The characters $\chi^{\prime G}, \chi$ running over all linear characters of $A$, form all the irreducible characters of $G$. See [1]. Every extension of $\chi$ to $K(L)$ is given by $\mu \chi^{\prime}$, where $\chi^{\prime}$ is a fixed extension of $\chi$ to $K(L)$ and $\mu$ is a linear character of $K(L)$ with $\operatorname{ker} \mu \supseteq A$. Note that if $L_{1}=\operatorname{ker} \chi^{\prime}$ then $L_{1} \cap A=L$ and $N\left(L_{1}\right) \subseteq N(L)$. Lemma 1 and the above results give

Corollary. Let $G$ be a finite group, $A$ a normal subgroup of $G, A$ and $G / A$ abelian, and let $\chi$ be a linear character of $A$. If some irreducible constituent of $\chi^{G}$ is real, then some element of $N(L)$ inverts all elements of $A / L$. If some irreducible constituent of $\chi^{G}$ is rational, then $N(L)$ induces the full automorphism group on $A / L$.

We omit the proof of the next result.

LEMMA 2. Let $U$ be a cyclic group of order $m$ and let $A=\operatorname{Aut}(U)$. For subgroups $V$ of $U$ let $B(V)=\mathbf{C}_{A}(U / V)$. Assume for every $V$ if $|U / V|=p, p$ an odd prime, then $B(V)$ has exponent dividing 2 , and if $|U / V|=2$ then $B(V)$ has exponent dividing 4 . Then either $m$ is a prime or $m$ divides 16,24 , or 40 .

3. The main result. We need the following

LEMMA 3. Let $A$ be a normal subgroup of finite group $G, G / A$ abelian, and let $\chi$ be a linear character of $A$ such that all the irreducible constituents of $\chi^{G}$ are real. Let $I=I_{G}(\chi)$, the inertia group of $\chi$ in $G$ and $L=\operatorname{ker} \chi$. Then

(a) $\left|I^{\prime} L / L\right| \leq 2$.

(b) If $I^{\prime} \subseteq \bar{L}$ then $\exp (I / A) \leq 2$.

(c) If $\chi^{2}=1_{A}$ then $\exp (I / A)$ divides 4

Proof. Since $G^{\prime \prime} \subseteq L$, we may assume $G$ to be metabelian. The elements of $I$ commute (modulo $L$ ) with the elements of $A$, and for any $y \in N(L)$ and $x \in I$, $y^{-1} x y \equiv x a_{x}(\bmod L), a_{x} \in A$. Thus for any $x, w \in I$ and $y \in N(L), y^{-1}[x, w] y \equiv$ $[x, w](\bmod L)$, where $[x, w]=x^{-1} w^{-1} x y$. Thus $I^{\prime} L / L$ is in the center of $N(L) / L$. Also $I^{\prime} L / L$ is cyclic since $A / L$ is cyclic. The realness of the constituents of $\chi^{G}$ implies that some element of $N(L)$ inverts $I^{\prime} L / L$. Thus $\left|I^{\prime} L / L\right| \leq 2$.

Now let $\chi^{\prime}$ be an extension of $\chi$ to $K(L) \subseteq I, \theta=\chi^{\prime I}$, an irreducible constituent of $\chi^{I}$, and $\mu$ be a linear character of $I$ with $\operatorname{ker} \mu \supseteq A$. If $\mu^{\prime}$ is an extension of $\mu$ to $G$ then $\mu \theta=\left(\mu_{K(L)} \chi^{\prime}\right)^{I}$ and $\mu^{\prime} \chi^{\prime G}=(\mu \theta)^{G}=\left(\mu_{K(L)} \chi^{\prime}\right)^{G}$, that is, $\mu \theta$ and $\mu^{\prime} \theta^{G}$ are constituents of $\chi^{I}$ and $\chi^{G}$ respectively. Now $\theta^{G}=\bar{\theta}^{G}$ and $(\mu \theta)^{G}=(\bar{\mu} \bar{\theta})^{G}$, bars denoting the complex conjugates. Thus

$$
(\bar{\mu} \bar{\theta})^{G}=\bar{\mu}^{\prime} \bar{\theta}^{G}=\bar{\mu}^{\prime} \theta^{G}=\left(\bar{\mu}^{\prime} \theta\right)^{G} \text { or }(\mu \theta)^{G}=(\bar{\mu} \theta)^{G} .
$$

From Isaacs $[5,6.11], \mu \theta=\bar{\mu} \theta$. Assuming $I^{\prime} \subseteq L$, we have $I=K(L), \theta$ linear, and hence $\mu=\bar{\mu}$ for every linear character $\mu$ of $I, \operatorname{ker} \mu \supseteq A$. Thus $\exp (I / A) \leq 2$.

Now assume $\chi^{2}=1_{A}$ and $\chi \neq 1_{A}$ (for if $\chi=1_{A}$ then $I^{\prime} \subseteq L$ ). Thus $|A / L|=2$ and $N(L)=I \supseteq K(L) \supseteq A$. If $y \in I$ and $k \in K(L)$, then $y^{-1} k y \equiv k a(\bmod L)$ for some $a \in A$, and hence $y^{2} \in K(L)$. Any extension of $\chi$ to $K(L)$ is given by $\mu \chi^{\prime}$ where $\mu$ is any linear character of $K(L), \operatorname{ker} \mu \supseteq A$, and $\chi^{\prime}$ is a fixed extension of $\chi$ to $K(L)$. Using the above, the realness of $\left(\mu \chi^{\prime}\right)^{\bar{G}}$ implies $\mu\left(k^{2}\right) \chi^{\prime}\left(k^{2}\right)= \pm 1$. Setting $\mu=1_{K(L)}$ we get $\chi^{\prime}\left(k^{2}\right)= \pm 1$. Thus $\mu\left(k^{2}\right)= \pm 1$ for every $\mu$ or $\exp (K(L) / A)$ divides 4. Now assume for some $k \in K(L), k$ of order 4 modulo $A$, and some $y \in I$, 
$y \notin K(L)$ and $y^{2} \equiv k(\bmod A)$. Then we may pick another $K(L)$, say $K_{1}(L)$, such that $y \in K_{1}(L)$ or $\exp \left(K_{1}(L) / A\right)=8$, a contradiction. Thus the above results imply $\exp (I / A)$ divides 4 completing the proof.

COROLlARY. Let $G$ be a finite metabelian group having all nonlinear irreducible characters real and for any subgroup $H$ of $G^{\prime}, G^{\prime} / H \neq 1$, let $t(H)=$ $\exp \left(\mathbf{C}_{G}\left(G^{\prime} / H\right) / G^{\prime}\right)$. Then $t(H)$ divides 4 if $\left|G^{\prime} / H\right|$ is a power of 2 and $t(H) \leq 2$ otherwise.

Proof. If $G^{\prime} / H \neq 1$, then $G^{\prime}$ has a linear character $\chi, H \subseteq \operatorname{ker} \chi \subset G^{\prime}$ and $\left|G^{\prime} / \operatorname{ker} \chi\right|$ a prime. The result follows from the above lemma.

THEOREM 1. Let $G$ be a finite metabelian group with all irreducible nonlinear characters rational and let $m=\exp \left(G^{\prime}\right)$. Then either $m$ is a prime or $m$ divides 16 , 24 , or 40.

Proof. Let $\chi$ be a linear character of $G^{\prime}, L=\operatorname{ker} \chi,\left|G^{\prime} / L\right|=m$. Then $N(L)$ induces the full automorphism group on $U=G^{\prime} / L$. Let $p \mid m, p$ a prime, and let $\lambda=\chi^{m / p}$. Then $S=\operatorname{ker} \lambda \supseteq L$ and letting $V=S / L$ we have $|U / V|=p$. Let $I=I_{G}(\lambda)$ then $I \cap N(L)=\mathbf{C}_{N(L)}(U / V)$.

Assume $p>2$. From Lemma 3(a), $\left|I^{\prime} S / S\right| \leq 2$, and hence $I^{\prime} \subseteq S$. Thus Lemma 3(b) implies $\exp \left(I / G^{\prime}\right) \leq 2$. If $p=2$, then $\lambda^{2}=1_{G^{\prime}}$, and from Lemma 3(c), $\exp \left(I / G^{\prime}\right)$ divides 4. Now $N(L) / \mathbf{C}_{N(L)}(U) \simeq \operatorname{Aut}(U)$ and thus $I \cap N(L) / \mathbf{C}_{N(L)}(U)$ $\simeq B(V)$ in the notation of Lemma 2. Since $\mathbf{C}_{N(L)}(U) \supseteq G^{\prime}$, we have $\exp (B(V)) \leq$ 2 if $|U / V|=p$, an odd prime, and $\exp (B(V))$ divides 4 if $|U / V|=2$. Now applying Lemma 2, we have $m$ a prime or $m$ dividing 16,24 , or 40 . The proof is complete.

If the linear characters of a finite group $G$ are rational, then $G / G^{\prime}$ is an elementary abelian 2-group. The above results give

COROLLARY. The order of a finite metabelian group with all characters rational is of the form $2^{t} 3^{s}$.

We give an example of a metabelian group $G$ with $\exp \left(G^{\prime}\right)=24$ and all nonlinear irreducible characters rational.

Let $\langle a, b\rangle$ and $\langle x, y, z\rangle$ be abelian groups with $a^{8}=b^{8}=1$ and $x^{2}=y^{2}=z^{2}=$ 1 , and let $G_{1}=\langle a, b\rangle \circ\langle x, y, z\rangle$, the semidirect product, with the action of $\langle x, y, z\rangle$ on $\langle a, b\rangle$ defined by

$$
a^{x}=a^{-1}, \quad b^{x}=b^{-1}, \quad a^{y}=a^{5}, \quad b^{y}=b^{5}, \quad a^{z}=b, \quad b^{z}=a .
$$

Then $G_{1}^{\prime}=\left\langle a^{-1} b, a^{2}\right\rangle$ and $\exp \left(G_{1}^{\prime}\right)=8$. It is easy to show that all the irreducible characters of $G_{1}$ are rational. Since all the irreducible characters of the dihedral group $D_{3}$ of order 6 are rational, the group $G=G_{1} \times D_{3}$ has all irreducible characters rational and $\exp \left(G^{\prime}\right)=24$.

The group $G_{2}=\langle a, b, x\rangle$ with defining relations $a^{2}=b^{4}=x^{2}=1, a b=b a$, $a x=x a$, and $x^{-1} b x=a b$ has all nonlinear irreducible characters rational and two linear characters complex. It is not known if metabelian groups $G$ with $\exp \left(G^{\prime}\right)=$ 16 or 40 exist that have all nonlinear irreducible characters rational.

4. Cyclic commutator group. We prove

THEOREM 2. Let $G$ be a finite metabelian group with all nonlinear irreducible characters rational and assume the commutator group $G^{\prime}$ is cyclic. Then the order of $G^{\prime}$ is a prime or divides 12 . 
Proof. From Theorem 1, if $\left|G^{\prime}\right|$ is odd then $\left|G^{\prime}\right|$ is a prime. Thus we assume $\left|G^{\prime}\right|$ is even and prove our result by taking the case when $\left|G^{\prime}\right|$ is a power of 2 , and then the case when $\left|G^{\prime}\right|$ is even but not a power of 2 .

Assume that $G^{\prime}=\langle a\rangle$ is cyclic of order $2^{s}$. Theorem 1 implies that $s \leq 4$. We shall prove that $s \leq 2$ by studying $\exp \left(K(1) / G^{\prime}\right)$ which from Lemma 3 divides 4 . Here $K(1)=K(\langle 1\rangle)$ is as in the notations of $\S 2$.

Assuming for some $K(1)$ the exponent of $K(1) / G^{\prime}$ is 4, we get the following two cases.

(i) There is $b$ in some $K(1),\left|b G^{\prime}\right|_{0}=4$ and $b^{4}=1$.

(ii) There is $b$ in some $K(1),\left|b G^{\prime}\right|_{0}=4$ and $b^{4}=a^{2}$.

Note that if $b^{4}=a$, then some nonlinear irreducible character of $G$ is not real, and if $b^{4}=a^{2^{t}}, t>1$, then setting $b_{0}=b^{-1} a^{2^{t-2}}$, we have $b_{0}^{4}=1$, which is case (i).

Assuming for every $K(1), K(1) / G^{\prime}$ is of exponent $\leq 2$, we get two additional cases.

(iii) $K(1) / G^{\prime}$ is of exponent 2 for every $K(1)$, and there is $c$ in some $K(1)$, $\left|c G^{\prime}\right|_{0}=2$ and $c^{2}=a$.

(iv) For every $K(1), K(1) / G^{\prime}$ is of exponent dividing 2 and $K(1)$ splits over $G^{\prime}$.

Note that if $c^{2}=a^{2^{t}}, t>0$, then setting $c_{0}=c^{-1} a^{2^{t-1}}$ gives $c_{0}^{2}$, which is case (iv). Also (iv) includes the case where $K(1)=G^{\prime}$.

We shall prove our result by studying separately each of the above four cases.

Case (i). Let $L=\left\langle a^{2}\right\rangle$ and pick $K(L) \supseteq\langle a, c\rangle$. Let $\chi$ be a linear character of $K(L)$ such that $\chi(b)=\sqrt{-1}, S \cap G^{\prime}=L$, where $S=\operatorname{ker} \chi$. Since $\chi^{G}$ is rational, we have $z \in N(S)$ such that $\chi\left(z^{-1} b z\right)=\chi\left(b^{-1}\right)=-\sqrt{-1}$. But $b^{-1} z^{-1} b z$ is in $G^{\prime}=\langle a\rangle$ implying $z^{-1} b z=b a^{f}, f$ an odd integer. Thus $\left(b a^{f}\right)^{4}=1$ or $a^{4 f}=1$ and $s \leq 2$.

Case (ii). Consider $K(1) \supseteq\langle a, b\rangle$ and let $\chi$ be a linear character of $K(1)$ with $S=\operatorname{ker} \chi$ and $S \cap G^{\prime}=1$. Then $K(1) / S=\langle b S\rangle$ and $\chi(b)^{4}=\chi\left(a^{2}\right)$. Assume $\chi(b)^{2}=\chi(a)$. (The other choice for $\chi$ is given by $\chi(b)^{2}=-\chi(a)$.) Since $\chi^{G}$ is rational we have $N(S) / K(1)=\langle y K(1), z K(1)\rangle$ such that $y^{-1} b y \equiv b^{5}(\bmod S)$ and $z^{-1} b z \equiv b^{-1}(\bmod S)$. Since $|b S|_{0}=2^{s+1}$ we have $|y K(1)|_{0}=2^{s-1}$ or $\left|y G^{\prime}\right|_{0}=$ $2^{s-1}, 2^{s}$, or $2^{s+1}$. But since $b^{-1} y^{-1} b y \equiv b^{4} \equiv a^{2}(\bmod S)$ and $S \cap G^{\prime}=1$, we have $y^{-1} b y=b a^{2}$. Let $L_{1}=\left\langle a^{2}\right\rangle$; then $y^{-1} b y \equiv b\left(\bmod L_{1}\right)$, and hence we may pick $K\left(L_{1}\right) \supseteq\langle a, b, y\rangle$, not necessarily containing $K(1)$. Thus $\left|y G^{\prime}\right|_{0}$ divides 4 or $s \leq 3$. Assume $s=3$, and let $y$ be as above. Then $y^{4}=1$ or $y^{4}=a^{2}$ or $y^{4}=a^{4}$. If $y^{4}=a^{2}$ then by letting $y_{0}=y b$ we get $y_{0}^{4}=1$. If $y^{4}=a^{4}$ then by letting $y_{1}=y a$ we get $y_{1}^{4}=1$. That is, we may assume $y^{4}=1$. Let $\chi_{1}$ be a linear character of $K\left(L_{1}\right), S_{1}=\operatorname{ker} \chi_{1}, S_{1} \cap G^{\prime}=\left\langle a^{2}\right\rangle$, and $\chi_{1}(y)=\sqrt{-1}$. Then there is $w \in N\left(S_{1}\right)$ such that $w^{-1} y w=y a^{f}, f$ an odd integer. But then $\left(y a^{f}\right)^{4}=a^{4 f} \neq 1$, a contradiction since $y^{4}=1$. Thus $s \leq 2$.

Case (iii). With slight variations, the proof of this case is similar to case (ii) above in which $c$ replaces $b$.

Case (iv). Assume every $K(1)$ can be written as $\left\langle a, c_{1}, \ldots, c_{t}\right\rangle$ with $a^{2^{\circ}}=c_{i}^{2}=1$, $i=1, \ldots, t$. If $s=4$ then by a method similar to case (ii), where $s=3$, in which $a$ replaces $b$, we get a likewise contradiction. Thus assume $s=3$.

Since $G^{\prime}=\langle a\rangle$, there are $g$ and $h$ in $G$ such that $g^{-1} h^{-1} g h=a^{e}, e$ odd. (Without loss of generality we may take $e=1$.) Thus $h^{-1} g h=g a$. We also have 
$g^{-1} a g=a^{\tau}$ and $h^{-1} a h=a^{\sigma}, \tau$ and $\sigma$ odd integers. First assume $\tau=1$. Then we may pick $K(1)$ such that $g \in K(1)$ and hence either $g^{2}=1$ or $\left(g a^{\lambda}\right)^{2}=1$, for some $\lambda$. Exchanging $g_{0}=g a^{\lambda}$ for $g$ gives $h^{-1} g_{0} h=g_{0} a^{f}, f$ odd. But $\left(g_{0} a^{f}\right)^{2} \neq 1$, a contradiction. Thus $\tau \neq 1$. Similarly $\sigma \neq 1$. Thus $\tau$ and $\sigma$ may take the values 3,5 , or $7(\bmod 8)$. If $\tau=\sigma$ then letting $g_{0}=g h$ we have $g_{0} a=a g_{0}$ and get a contradiction as above. Thus $\tau \neq \sigma$, and hence one of $\tau, \sigma$, or $\tau \sigma$ takes the value $5(\bmod 8)$. Without loss of generality we assume $\tau=5$ and $\sigma=-1 \equiv 7(\bmod$ 8). That is, we have $g^{-1} a g=a^{5}$ and $h^{-1} a h=a^{-1}$. Since $h^{-1} g h=g a$ we have $(g a)^{2}=g^{2} a^{6}$ and $(g a)^{4}=g^{4} a^{4}$.

Let $L=\left\langle a^{4}\right\rangle$. Then $g^{-1} a g \equiv a(\bmod L)$, and thus we may pick $K(L)$ such that $g \in K(L)$. This implies that either $\left|g G^{\prime}\right|_{0}=2$ or $\left|g G^{\prime}\right|_{0}=4$. Assume $\left|g G^{\prime}\right|_{0}=2$; then we have $g^{2}=1, g^{2}=a^{2}$, or $g^{2}=a^{4}$. Then the orders of $h^{-1} g h=g a$ and of $g$ are not equal, a contradiction. Thus $\left|g G^{\prime}\right|_{0}=4$ which implies $g^{4}=1, g^{4}=a^{4}$, or $g^{4}=a^{2}$. Again in the first two cases, the orders of $h^{-1} g h=g a$ and of $g$ are not equal, a contradiction. This leaves us with the only case $g^{4}=a^{2}$.

Let $\chi$ be a linear character of $K(L) \supseteq\langle a, b\rangle, S=\operatorname{ker} \chi, S \cap G^{\prime}=L$, and assume $\chi(g)^{2}=\chi(a)$. Then $K(L) / S=\langle g S\rangle$ and thus $N(S) / K(L)=\langle y K(L), z K(L)\rangle$ with $y^{-1} g y \equiv g^{5}(\bmod S)$ and $z^{-1} g z \equiv g^{-1}\left(\bmod S^{\prime}\right)$. Thus $y^{-1} g y \equiv g a^{2}(\bmod L)$ and $z^{-1} g z \equiv g a^{-1}(\bmod L)$. Since $y^{-1} a y \equiv a(\bmod L)$, it follows that $y^{-1} a y=a$ or $y^{-1} a y=a^{5}$. If $y^{-1} a y=a^{5}$, then letting $y_{0}=y g$, we get $y_{0}^{-1} a y_{0}=a$. Thus, we may assume $y^{-1} a y=a$. Hence we may pick some $K(1)$ such that $y \in K(1)$. But the assumptions on $K(1)$ imply that either $y^{2}=1$ or $\left(y a^{\lambda}\right)^{2}=1$ for some $\lambda$. Without loss of generality, we may assume $y^{2}=1$. Since $y^{-1} g y \equiv g a^{2}(\bmod$ $L$ ), we have $y^{-1} g y=g a^{2}$ or $g a^{-2}$. These give $g^{-1} y g=y a^{-2}$ or $y a^{2}$. But then $\left(g^{-1} y g\right)^{2} \neq 1$, a contradiction. This completes the proof of case (iv) and the proof of the first part of the theorem.

Now assume $\left|G^{\prime}\right|$ is even but not a power of 2 . From Theorem 1 only 3 or 5 may divide $\left|G^{\prime}\right|$. We make a slightly more general assumption, that is, $G^{\prime}$ has a cyclic 5-Sylow subgroup and a not necessarily cyclic 2-Sylow subgroup, and then arrive at contradictions.

Let $G^{\prime}=A \times B, A$ an abelian 2-group, $B=\langle b\rangle,|B|=5$. Let $L$ be a (fixed) subgroup of $A,|A / L|=2, A / L=\langle a L\rangle$ and $B=\langle b\rangle$. Then $G^{\prime} / L=\langle a b L\rangle$ is of order 10. Assuming all the nonlinear irreducible characters to be rational we have $\exp \left(K(L) / G^{\prime}\right) \leq 2$.

We have the following two cases:

(v) For every $K(L), K(L) / L$ splits over $G^{\prime} / L$, i.e., $K(L) / L=G^{\prime} / L \times E / L, E / L$ an elementary abelian 2-group.

(vi) There exists some $c$ in some $K(L)$ such that $c^{2} \equiv a(\bmod L)$.

Case (v). Assume (v) and fix $K(L)$. Let $\chi$ be a linear character of $K(L), \operatorname{ker} \chi=$ $L_{1}, L_{1} \cap G^{\prime}=L$. Then $N\left(L_{1}\right) \subseteq N(L)$ and $N\left(L_{1}\right) / K(L) \simeq \operatorname{Aut}\left\langle K(L) / L_{1}\right\rangle$, $N\left(L_{1}\right) / K(L)=\langle y K(L)\rangle$ is of order 4 , and $y^{-1} a y \equiv a(\bmod L), y^{-1} b y \equiv b^{2}(\bmod$ $L)$ and $y^{-1} z y \equiv z(\bmod L)$ for all $z \in E$. Now let $\bar{L}=\langle L, B\rangle=L \times B$. Since $y$ commutes $(\bmod \bar{L})$ with all elements of $K(L)$, we choose $K(\bar{L}) \supseteq\langle K(L), y\rangle=$ $N\left(L_{1}\right)$. It could easily be shown that $K(\bar{L})=N\left(L_{1}\right)$ and $N(\bar{L})=N(L)$. If $y^{4} \equiv a$ $(\bmod \bar{L})$, then some nonlinear character of $N(\bar{L})($ and hence of $G$ ) is not real. Since $y^{2} \notin G^{\prime}$ we have $y^{4} \equiv 1(\bmod \bar{L})$, and hence we may assume $y^{4} \equiv 1(\bmod L)$. Thus $K(\bar{L}) / \bar{L}$ splits over $G^{\prime} / \bar{L}$, i.e., $K(\bar{L}) / \bar{L}=G^{\prime} / \bar{L} \times\langle E, y, b\rangle / \bar{L}$. 
Let $\theta$ be a linear character of $K(\bar{L})$ with $\bar{L}_{1}=\operatorname{ker} \theta, \bar{L}_{1} \cap G^{\prime}=\bar{L}$, and $\theta(y)=$ $\sqrt{-1}$. Let $x \in N\left(\bar{L}_{1}\right)$; then $x \in N(\bar{L})=N(L)$ and $x^{-1} z x \equiv z(\bmod \bar{L})$ for all $z \in E$. Thus $x^{-1} z x \equiv z(\bmod L)$ for all $z \in E$, and hence $x \in N\left(L_{1}\right)=K(\bar{L})$. Thus $N\left(\bar{L}_{1}\right)=K(\bar{L})$, and since $\theta$ is not real, $\theta^{G}$ is not real, a contradiction.

Case (vi). In this case we may assume $K(L) / L=\langle c L\rangle \times B \times E / L, E / L$ an elementary abelian 2-group, and $G^{\prime} / L=\left\langle c^{2}, B\right\rangle / L$. If $\chi$ is a linear character of $K(L), \operatorname{ker} \chi=L_{1}, L_{1} \cap G^{\prime}=L$, and $\chi(c)=\sqrt{-1}$, then $K(L) / L_{1}=\left\langle c b L_{1}\right\rangle$, of order 20, and $N\left(L_{1}\right) / K(L)=\langle x K(L), y K(L)\rangle$ with

$$
\begin{gathered}
x^{-1} c x \equiv c^{-1}, \quad y^{-1} b y \equiv b^{2}, \quad x^{-1} b x \equiv b, \\
y^{-1} c y \equiv c, \quad x^{-1} z x \equiv y^{-1} z y \equiv z,
\end{gathered}
$$

with all congruences modulo $L$ and $z \in E$. Let $\bar{L}=\langle L, B\rangle$ and pick $K(\bar{L}) \supseteq$ $\langle K(L), y\rangle$. Then $K(\bar{L})=\langle K(L), y\rangle$. Let $\theta$ be a linear character of $K(\bar{L}), \bar{L}_{1}=\operatorname{ker} \theta$ and $\bar{L}_{1} \cap G^{\prime}=\bar{L}$. If $y^{4} \equiv a(\bmod \bar{L})$, then $\theta^{G}$ is not real. Assume $y^{4} \equiv 1(\bmod \bar{L})$ and hence assume $y^{4} \equiv 1(\bmod L)$. Then $K(\bar{L}) \subseteq N\left(\bar{L}_{1}\right) \subseteq N\left(L_{1}\right)=\langle K(\bar{L}), x\rangle$. If $x^{-1} y x \equiv y(\bmod L)$, then let $\theta(c)=\theta(y)=\sqrt{-1}$. Then $x^{-1}\left(c^{-1} y\right) x \equiv c y$ $(\bmod L)$, and since $\theta\left(c^{-1} y\right)=1$ and $\theta(c y)=-1$, we have $x \notin N\left(\bar{L}_{1}\right)$. Thus $N\left(\bar{L}_{1}\right)=k(\bar{L})$ and $\theta^{G}$ is not real. If $x^{-1} y x \equiv y a(\bmod L)$ then let $\theta(y)=1$. Since $\theta(y a)=-1$, again $N\left(\bar{L}_{1}\right)=K(\bar{L})$ and $\theta^{G}$ is not real. These contradictions complete the proof of this part and proof of the theorem.

The above proof also gives

COROLlARY. Assume all the nonlinear irreducible characters of the metabelian group $G$ are rational and $G^{\prime}$ is of even order. If the $p$-Sylow subgroup of $G^{\prime}$ is cyclic where $p$ is an odd prime, then $p=3$.

We conclude with

COROLlARY. Assume every nonlinear irreducible character of $G$ is rational and $G^{\prime}$ is cyclic. If $\left|G^{\prime}\right|=p, p$ an odd prime, then $|G|=2^{t} p(p-1) ;$ if $\left|G^{\prime}\right|=2$ or 4 then $|G|=2^{t}$, and if $\left|G^{\prime}\right|=6$ or 12 then $|G|=2^{t} \cdot 3$.

ACKNOWLEDGEMENTS. The author would like to thank the referee for his numerous suggestions specially those involving Lemmas 2 and 3 and Theorem 1.

\section{BIBLIOGRAPHY}

1. B. G. Basmaji, Monomial representations and metabelian groups, Nagoya Math. J. 35 (1969), 99-107. MR 39 \#5709.

2. - Representations of metabelian groups realizable in the real field, Trans. Amer. Math. Soc. 169 (1972), 389-399. MR 46 \#9153.

3. C. W. Curtis and I. Reiner, Representation theory of finite groups and associative algebras, Pure and Appl. Math., vol. XI, Interscience, New York, 1962. MR 26 \#2519.

4. R. Gow, Groups whose characters are rational-valued, J. Algebra 40 (1976), 286-299. MR 53 \#13372.

5. I. M. Isaacs, Character theory of finite groups, Academic Press, New York, 1976. MR 57 \#417.

6. F. M. Markel, Groups with many conjugate elements, J. Algebra 26 (1973), 69-74. MR 48 \#8624.

7. A. L. Vishnevetskij, Finite groups with rational characters, Dokl. Akad. Nauk Ukrain. SSR, Ser. A (1977), no. 10, 876-878, 958. (Russian) MR 57 \#9822.

Department of Mathematics, California State University, los ANgeles, CALIFORNIA 90032 